\title{
Foreword
}

\section{Cardiac Anesthesia}

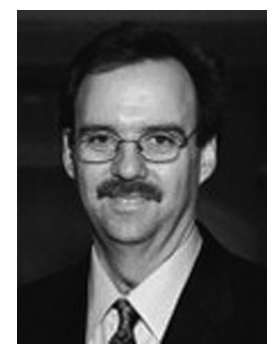

Lee A. Fleisher, MD, FACC, FAHA

Consulting Editor

Anesthesia for patients undergoing cardiac procedures has advanced greatly in the past several decades. It is one of the areas in which anesthesiologists have partnered closely with their surgical colleagues to advance patient care. With the development of less invasive procedures, especially in the electrophysiology laboratory, our role in the care has increased. In this issue of Anesthesiology Clinics, the guest editor has assembled an outstanding group of investigators to discuss a wide range of procedures. Additionally, she has outlined advances in patient management leading to improvement in patient safety.

I was fortunate to recruit Colleen G. Koch, MD, MS, MBA as guest editor for this issue. Dr Koch received her Medical Degree from the University of Cincinnati College of Medicine followed by an anesthesia residency at Brigham and Women's Hospital. She also completed a Masters Degree in Clinical Research Design and Statistics from the University of Michigan School of Public Health and a Masters in Business Administration from Case Western Reserve University. She is currently Professor of Anesthesiology and Vice Chair of Research and Education Department of Cardiothoracic Anesthesia at the Cleveland Clinic and holds a Joint Appointment Quality and Patient Safety Institute, Research, and Education. She is also a member of the Board of Directors of the Society of Cardiovascular Anesthesiologists. She is therefore well qualified to assemble a great group of leaders within cardiovascular anesthesia to discuss these important topics.

\section{Lee A. Fleisher, MD, FACC, FAHA Perelman School of Medicine University of Pennsylvania Philadelphia, PA 19104, USA \\ E-mail address: lee.fleisher@uphs.upenn.edu}

